\title{
Overline: Education
}

\section{Personalized Medical Education: Reappraising Clinician-Scientist Training}

\author{
Gabriele C. DeLuca1 ${ }^{1}$ Pavel V. Ovseiko² and Alastair M. Buchan²,3
}

${ }^{1}$ Nuffield Department of Clinical Neurosciences and Oxford Medical School, University of Oxford, Oxford, OX3 9DU, UK.

${ }^{2}$ Radcliffe Department of Medicine, University of Oxford, OX3 9DU, Oxford, UK.

${ }_{3}^{3}$ Medical Sciences Division, University of Oxford, OX3 9DU, Oxford, UK

Corresponding author E-mail: alastair.buchan@medsci.ox.ac.uk

\begin{abstract}
Revitalizing the Oslerian ideal of the clinician-scientist-teacher may help in the training of the next generation of translational researchers.
\end{abstract}

William Osler's ideal of the clinician-scientist-teacher not only set standards for medical education on both sides of the Atlantic more than a century ago, but also holds solutions for the training of the next generation of translational researchers today. Having pioneered modern bedside teaching in Canada and the USA in the late 1800s, Osler was appointed Regius Professor of Medicine at the University of Oxford in 1905. Upon his arrival from Johns Hopkins to Oxford, he discovered that in England research and preclinical medical education in universities were dissociated from clinical practice and postgraduate training in hospitals. As Osler was convinced that future advances in medical education and patient care would come from research, he challenged the English medical establishment to integrate research into medical education and patient care under the auspices of a university professor: "The Professor has three duties---to see that the patients are well treated, to investigate disease, and to teach students and nurses" (1). He argued that great scientific discoveries came from "the pursuit of knowledge for its own sake", and that already in the early 20th century the hallmark of such discoveries was their translatability into practical applications(1). Thus, the enduring challenge in modern medicine is not only scientific innovation, but also translation of scientific discoveries into new therapies for the benefit of humanity.

\section{The challenges of a joint clinician-scientist training program}

Today, the challenges identified by Osler are particularly acute in the training of clinicianscientists, i.e. clinically qualified physicians substantially engaged in scientific research. Clinician-scientists can achieve the research skills necessary to engage in cutting-edge science through a variety of educational pathways. Joint MD-PhD programs are widely used in Canada and the USA, but are rare in the UK and continental Europe, where medical students and postgraduate trainees (i.e. residents) follow various functionally equivalent training pathways in the pursuit of a clinician-scientist career. These range from extensive involvement in research projects and completion of masters-level research 
degrees during medical training to dedicated research fellowships and clinician-scientist development training after graduation from medical school.

Regardless of country and training pathway, trainees invested in developing both clinical and research expertise face daunting challenges. Due to the increased complexity of modern science and patient care, students in joint MD-PhD programs in North America and their functional equivalents around the world take up to eight years to complete both degrees. This results in high risks for students as some drop out during their training, e.g. up to $27 \%$ of MD-PhD matriculants in the USA (2). Those aspiring clinician-scientists without financial support who persevere can endure mounting student debt and a poor work-life balance. When new clinician-scientists eventually start their independent research careers, they do not get to enjoy a grace period of time, money, and energy necessary to develop new ideas, as they are immediately caught up in the race to publish, the struggle to survive from grant to grant, and the ever increasing demands of maintaining medical accreditation.

To add further complexity, obtaining both an MD and $\mathrm{PhD}$ does not necessarily a clinicianscientist make. Medical and research degrees differ significantly, as they are predicated on fundamentally different approaches. Whereas medical education seeks to provide a platform to teach students to enhance patient benefit while minimizing clinical risk, research degrees aim to attain the same goal through taking scientific risks. Integrated exposure to these dichotomous (but complementary) ways of thinking is lacking, as students pursue medical and research degrees in separated blocks of time. Students spend too much of their time learning at the bench during their scientific research training rather than conducting clinical research or practicing clinical skills at the patient's bedside. The reality is that time for research in medical school is sparse such that medical students on clinical rotations are often pushed into becoming passive observers of rather than active participants in the cutting-edge scientific process. This is further compounded by the growing schism between clinical and research departments in medical schools and postgraduate training programs (3). Many medical school labs that previously focused on clinical teaching and clinical investigations have moved out of hospitals to purpose-built institutes and research centers that focus on basic sciences. It is thus not surprising that, increasingly, clinician-scientists struggle to capitalize on their clinical and research expertise to perform innovative translational research.

Despite the creation and spread of joint MD-PhD degrees in North America in the 1960s, the above mentioned challenges have adversely affected the ability of clinician-scientists to innovate. This is best illustrated by the training pathways of Nobel Laureates in Physiology or Medicine, the Nobel Prize being a robust marker of scientific innovationBy examining the biographies of 210 Nobel Laureates in Physiology or Medicine awarded since 1901 and posted on the Nobel Prize website (4), we found a steady decline in the proportion of clinician-scientists awarded the Nobel Prize in Physiology or Medicine (Fig.1). Clinician-scientists accounted for $73 \%$ of Nobel Laureates in Physiology or Medicine from 1901 to 1960; but from 1961 to 2015 their proportion dropped to 42\%. In contrast, the proportion of Nobel Laureates in Physiology or Medicine who are nonclinically trained scientists has increased from $27 \%$ in 1901-1960 to 58\% in 1961-2015. The shift in predominant career pathways of Nobel Laureates in Physiology or Medicine from clinician-scientists to non-clinically trained scientists, in part, reflects the shift of the most awarded areas from human diseases until 1960 to basic molecular biology and genetics after 1960. The downside of this shift is that most basic science discoveries have 
yet to be translated into therapies and improved patient care, while the burden of chronic disease associated with the $21^{\text {st }}$ century's aging population is escalating.

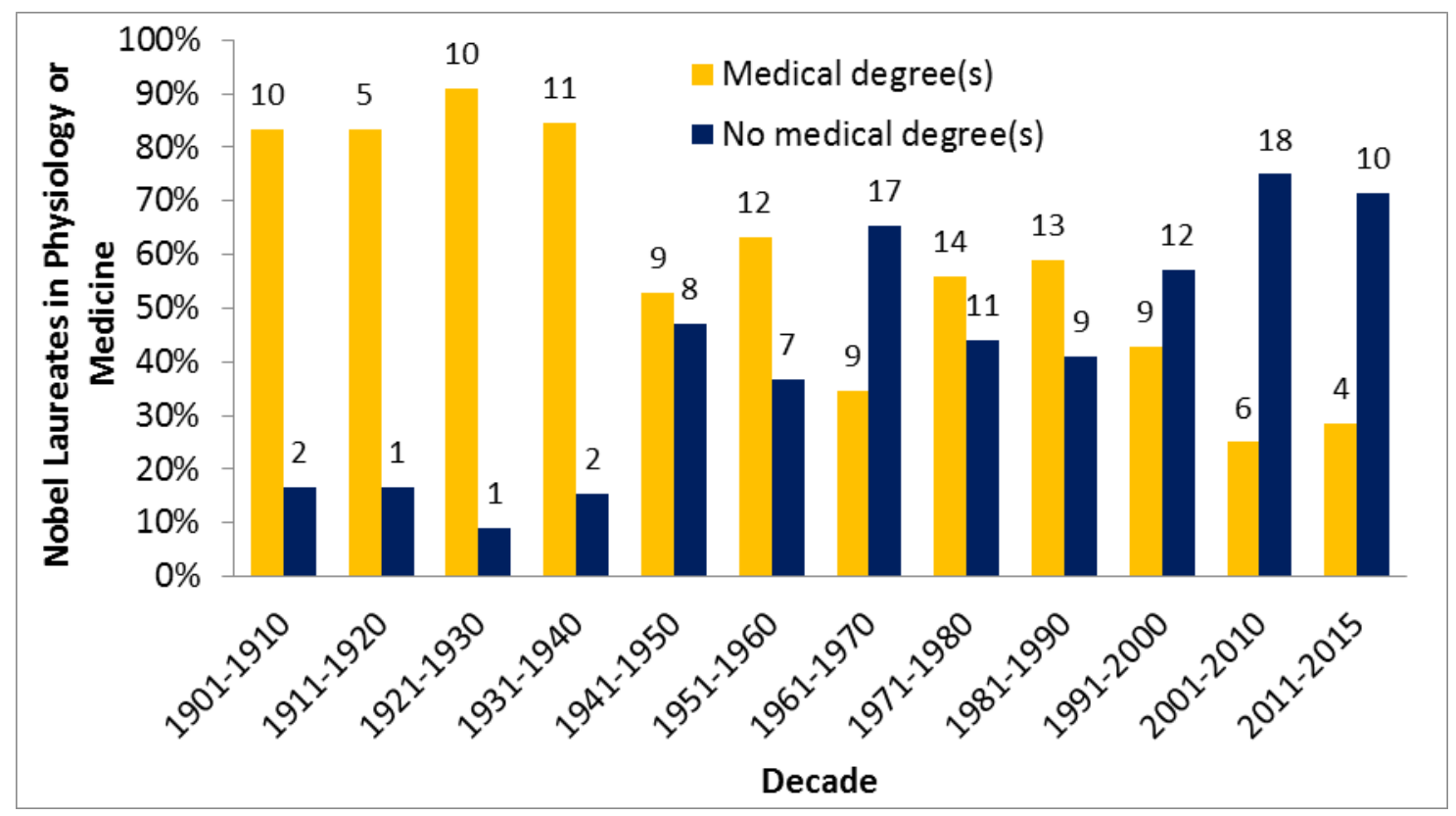

Fig.1. Nobel Laureates in Physiology or Medicine by medical degree, 1901-2015. A steady decline in the proportion of clinician-scientists awarded the Nobel Prize in Physiology or Medicine has led to the shift in predominant career pathways of Nobel Laureates in Physiology or Medicine from clinician-scientists to non-clinically trained scientists. Bars indicate proportions; figures above the bars indicate absolute numbers; no Nobel Prize was awarded in 1915-1918, 1921, 1925, 1940-1942. Source: Nobel Laureates' biographies on the Nobel Prize website (4).

\section{Integration of clinical and research education}

Following a crisis in the numbers of clinician-scientists in the UK in the early 2000s, government, industry, medical research charities, and universities, recognized the need to strengthen clinical research and innovation in the National Health Service (NHS) for the benefit of patients. To achieve this, a wide group of stakeholders chaired by Mark Walport, then Director of the Wellcome Trust, set out to modernize medical careers in the UK (5), and the government created the National Institute for Health Research (NIHR) (6). Within this framework, Oxford academic and healthcare partners established the Oxford University Clinical Academic Graduate School (OUCAGS) (7) aimed at better integrating the exposure to clinical practice, research, and teaching as advocated by Osler. In addition to traditional masters and doctoral programs for aspiring clinician-scientists, OUCAGS offers new NIHR-funded training pathways based on Walport's recommendations: academic clinical fellowships allowing trainees to undertake $25 \%$ research and $75 \%$ clinical training over 3-4 years, usually, to develop a proposal for a doctorate; and clinical lectureships allowing trainees to undertake $50 \%$ research and $50 \%$ clinical training over 4 years, usually, to conduct postdoctoral research and teaching. The faculty and trainees are particularly enthusiastic about these new pathways because they attempt to bridge the organizational divide between the university and the hospital and to enable trainees to develop and pursue their own line of research. Moreover, funding for academic clinical fellowship and clinical lectureship posts comes predominantly from NIHR, reflecting the societal importance of translational clinical research. 
Whilst the implementation of NIHR and OUCAGS have helped narrow the gap between clinician and scientist, it has highlighted the pressing need to develop more integrated, dynamic, and flexible clinician-scientist training programs that span the entire continuum of undergraduate and postgraduate education and training. We believe that to achieve this we need to revitalize the Oslerian ideal of the clinician-scientist-teacher. First, the integration of clinical training and exposure to research needs to be strengthened. Second, teaching and mentorship need to be equal partners with research and patient care. Third, scientifically innovative translational research for patient benefit needs to become the raison d'être of clinician-scientist training and careers. It is time for a seismic shift in the way the next generation of clinician-scientists are educated.

Exposure to and involvement in clinical translational research throughout medical school and postgraduate specialty training is a key tenet. Trainees interested in a clinicianscientist career should be given an opportunity to undertake a simultaneous parallel research path during medical school and residency. Protected time would be given to pursue short research projects relevant to curricular modules of interest throughout the medical school course. For example, a student learning the fundamentals of cardiology in the classroom or at the bedside could take a short detour from their "standard" medical course to pursue a research project in that field. The duration of dedicated research project time would need to be flexible (e.g. 2-6 months) to accommodate project goals and demands. This would facilitate the introduction and consolidation of core medical principles while contributing to cutting-edge science.

Trainees would be expected to devise a personalized curriculum to enable them to weave research in and out of the regular medical school and residency curriculum. In so doing, contextual knowledge and real world experiences (i.e. clinical and scientific) would be married through a problem-solving based approach. Time allotted to the acquisition of core medical knowledge, bedside apprenticeship, and research training would be dependent on competency-based outcomes and not restricted to time-fixed rotations. The duration of research exposure throughout the degree course would vary according to the trainees needs and interests, with the candidate potentially being awarded a higher research degree (e.g. MSc or PhD) arising from their scientific contributions. This system would provide a unique platform to arm the emerging clinician-scientist with the skills needed to be adequately trained in providing an excellent clinical service at the bedside while taking scientific risks at the bench for the betterment of patient care.

\section{Value of teaching and mentorship}

Critical to the success of such a system would be close mentorship of trainees by dedicated, established clinician-scientists, similar to the Oslerian ideal. The personalized nature of the proposed training program would require the close guidance and regular critical appraisal of a trainee's performance from an assigned mentor, who would oversee their clinical and research activities. Enthusiastic clinical researchers from a range of specialties would supervise research projects while interacting regularly with the trainee's mentor to discuss progress and to mobilize support. The time intensive nature of such an "apprenticeship" would necessarily limit the number of trainees selected on a competitive basis to pursue this path (i.e. less than $10 \%$ of a medical school class due to mentorship and resource limitations). Further, institutions would be expected to protect the time of talented clinician-scientist educators and mentors so that they may engage adequately with clinician-scientist candidates. The stale but pervasive view that educators are failed clinicians or scientists requires dismantling, and the integral role of 
teaching as part of clinician-scientist training demands resurrection. The means by which institutions evaluate "academic contribution" needs careful reconsideration.

\section{Importance of innovation and translation}

Scientifically innovative translational research for patient benefit needs to become the raison d'être of clinician-scientist training and careers. To achieve this goal, clinicianscientist training needs to be recalibrated from molding the brightest clinicians into basic scientists to training clinicians to translate basic science discoveries into health benefits for local and global patient communities. Therefore, it would be important to maximize involvement of trainees in clinical and research organizations that are funded by taxpayers to focus on impactful translational research. For example, institutions with Clinical and Translational Science Awards (CTSAs) in the USA and Biomedical Research Centres (BRCs) in the UK could offer prime environments and partial resources for clinician-scientist training. Given that some of the greatest health benefits can be achieved at the level of health systems and global populations, clinician-scientist programs would also need to include medical humanities, social sciences, public health policy, health services research, and leadership and management training to change suboptimal health systems and social structures.

Finally, government and society as a whole need to encourage and recognize impactful translational research. Funders of health research should encourage translational research by basing funding awards on the impact of the research on patients rather than according to academic outputs. For example, the current Research Excellence Framework exercise in the UK seeks to allocate $20 \%$ of taxpayers' funding in medicine and all other academic disciplines to universities based on the societal and economic impact of their research. It is also important for society to raise the profile of translational research and recognize achievements of clinician-scientists. Prestigious scientific awards and prizes need to better balance recognition of innovative scientific discovery with impactful translational research leading to significant clinical benefit. Translational medicine has evolved sufficiently as a discipline to warrant a high profile award analogous to the Fields Medal or Nobel Prize.

\section{Conclusions}

We are at a critical juncture in the field of medicine and scientific discovery where the burden of chronic disease is escalating and the translational impact of research is falling further behind. By strengthening the integration between clinical training and exposure to research, enhancing the value of teaching and mentorship, and encouraging scientific innovation and translation for patient benefit, the next generation of clinician-scientists will be better poised to overcome the medical challenges of this century. In so doing, we stand to benefit from the humanistic value of their research, which would be deserving of the most prestigious of scientific awards.

\section{Acknowledgements}

Gabriele C. DeLuca and Alastair M. Buchan are supported by the NIHR Oxford Biomedical Research Centre. 


\section{References}

1. W. Osler, An address on the hospital unit in university work delivered before the Northumberland and Durham Medical Society. Lancet 177, 211-213 (1911).

2. D. B. Jeffe, D. A. Andriole, H. D. Wathington, R. H. Tai, Educational outcomes for students enrolled in MD-PhD programs at medical school matriculation, 19952000: a national cohort study. Acad. Med. 89, 84-93 (2014).

3. A. M. Feldman, M. S. Runge, J. G. N. Garcia, A. H. Rubenstein, American medical education at a crossroads. Sci. Transl. Med. 7, $285 \mathrm{fs} 217$ (2015).

4. Noblelprize.org, All Nobel Prizes in Physiology or Medicine. (Nobel Media AB, http://www.nobelprize.org/nobel prizes/medicine/laureates/, 2014).

5. UK Clinical Research Collaboration, Modernising Medical Careers, Medically- and dentally-qualified academic staff: Recommendations for training the researchers and educators of the future. Report of the Academic Careers Sub-Committee of Modernising Medical Careers and the UK Clinical Research Collaboration Chaired by Dr Mark Walport, Director of The Wellcome Trust. (UK Clinical Research Collaboration, London, 2005).

6. Department of Health, Best research for best health: a new national health research strategy. (Department of Health, London, 2006).

7. K. Fleming, C. Pugh, D. Best, Academic postgraduate medical education---an Oxford view. Clin. Med. 14, 38-41 (2014). 